\title{
An Integrated Framework for Biometrics Security
}

\author{
Kashif lqbal \\ Department of Computing and the \\ Digital Environment, \\ Coventry University, UK \\ k.iqbal@coventry.ac.uk
}

\author{
Michael O. Odetayo \\ Department of Computing and the \\ Digital Environment, \\ Coventry University, UK \\ m.o.odetayo@coventry.ac.uk
}

\author{
Anne James \\ Department of Computing and the \\ Digital Environment, \\ Coventry University, UK \\ a.james@coventry.ac.uk
}

\begin{abstract}
Security methods based on biometric are gaining importance increasingly for the last few years. Such methods take into account humans' unique physical or behaviour traits that help to identify them based on their intrinsic characteristics. In this paper, we have proposed an integrated framework for image enhancement, face detection, feature extraction and face recognition. The proposed framework will be applied to images produced by surveillance cameras which usually produce low quality images.
\end{abstract}

\section{Keywords}

Biometrics security, image enhancement, face detection, feature extraction, face recognition.

\section{INTRODUCTION}

Biometrics security is concerned with identifying humans based on their unique physical and behavioural traits. By physical traits we mean the feature of human body such as fingerprint, DNA, Iris recognition, hand geometry and face recognition. Behavioural traits mean voice, gait and typing rhythm including digital signature. In these days, biometrics security measures are increasingly investigated and being used in several applications based on these traits. The main objective of such applications is to detect and recognize a human for security purposes [1]. The applications of biometric security are growing in public areas. Surveillance cameras are used to monitor traffic flow, threats, and suspicious activities in order to support government and lawenforcement agencies. Surveillance cameras play a vital role in security issues as they are widely deployed in public places to prevent or investigate crimes and to detect a suspicious person in order to make the environment more secure.

The main problem with surveillance images is that they are of low quality [2]. This makes the detection and recognition of objects on images captured by surveillance cameras difficult if not impossible. The quality of the surveillance videos is low due to several reasons such as: movement of objects; use of low resolution cameras and; environmental factors. The environmental factors include rainfall, fog and snow. These factors obscure object details and create noise which badly effect object detection and recognition [3]. It is therefore crucial to enhance the quality of surveillance images in order to use them for face detection and recognition purposes [4] [5].

In biometric security systems, face detection is the first and very crucial stage, as faces have to be detected from the input image before they can be further processed or recognised. In existing literature face recognition methods are mostly applied to passport quality pictures. Due to the high quality of these pictures, the systems are able to produce better and accurate results [6]. Unfortunately the existing methods are not applied to the surveillance images due to the poor quality of perception. In the existing research, the ambitious problem of biometric security from image enhancement perspective has not been fully addressed.

\section{LITERATURE REVIEW}

This section discusses the current literature on biometrics security. The objective of this section is to present the existing approaches and their perceived limitations. We are of the view that the existing approaches do not address the problem concerning the biometric security from image detection to image recognition through image enhancement.

[3] has proposed a framework to monitor the city traffic flow. The framework mainly focuses on the subtraction of background and shadow removal from the images/videos. Two background subtraction methods have been used for this purpose. They include deterministic and statistical methods. These methods use the adaptive background and per pixel Gaussian mixture in order to obtain the background information. The main drawback of this approach is that mostly it cannot detect the complete foreground objects. Also, the approach has not addressed the object recognition issues.

Few researchers have also enhanced the quality of images by improving the edges visibility. For example, [7] quite recently proposed a method for the recognition of car license number plate. The method is based on image enhancement technique which improves edge density for better object recognition. This research has enhanced the [8] work that was developed based on intensity variance of pixel intensities of local neighbourhood pixel in order to improve the contrast of car number plate region [7].

[9] have also proposed a method to enhance low resolution images and improve the edges visibility of surveillance images. The method is based on Weighted Markov Random Field Regularization. The method has been applied to enhance the edges and detect car number plates.

The focus of some approaches has been on object detection which can be used for object recognition. To detect the moving objects, there are few methods available such as optical flow, background subtraction and frame differencing [10] [11]. [10] have proposed a method based on Pixel Bit Mask Difference and History Update Values. The later method helps to reduce false detection. In order to detect the moving objects, an edge detection method was used. 
[12] have proposed an approach in order to detect and track the objects in a video stream. The approach relies on multiple factors such as motion, shape and colour of an object. These factors create the connectivity between the objects in order to track them. The method fragments the moving objects based on background and shadow for object tracking purposes. The method has not addressed the issues of face recognition.

In order to address the problem of low quality of images produced by surveillance cameras, [2] have developed an application, based on gradient method, to enhance the low resolution of surveillance images and videos. A gradient method is used to record the sequence of images at subpixel level in order to improve the resolution and to get the undistorted and sharp images. Another approach addresses the same problem of low resolution using a Genetic Algorithm. The approach is applied to obtain the best set of registration parameters which are used to determine the points using a spread function to improve the resolution of low quality images [13].

[5] have proposed a face recognition approach. The approach is applied to increase the resolution of surveillance images. Secondly, it uses the high resolution images for face recognition. To address the problem of resolution from both perspectives (low to high and high to low), the approach uses the Bilinear function along with the Gaussian Pyramid method. The Gaussian Pyramid method is used to increase the resolution (low to high) of an image and the Bilinear function is used to decrease the resolution (high to low). The Principal Component Analysis (PCA) and Linear Discriminant Analysis (LDA) methods are used for face recognition purposes [5].

Another important aspect related to the quality of image is to maintain uniform illumination throughout the image. Un-uniform illumination destroys the colours. [14] have proposed a method to restore the colours in highly illuminated images. This research helps to restore the colour information of high illuminated images where the colour information has been lost. The approach is based on the correction vectors method which is able to bring back the colours in the image in order to recognise the objects [14].

An integrated method for face recognition has been proposed by [15]. The integration is based on three methods: contrast enhancement, feather extraction and edge enhancement. The integrated method is able to address three basic issues. The problem of low illumination is addressed by applying the local contrast enhancement method. Secondly, the selection of feather extraction is carried out using adaptive feather extraction method. Thirdly, support vector machine based technique is used for classification.

[16] have proposed an illumination normalization model (INM) for face recognition. This model is based on two stages. At first stage the image is decomposed into high and low frequencies. Then the illuminated image is generated by low frequency. In second stage the noise is removed and contrast enhancement is carried out. For these experiments passport quality images were used. Such images are normally considered of high quality.

[17] have proposed a biometric method which is based on two levels. At the feature level they used modified Sparse Random Matrix for feature extraction. In the second level they used approximation step function for template transformation in order to minimize the error rate. Before applying these two levels of methodology they perform a preprocessing stage and in this stage they have used histogram equalisation method for image enhancement in order to get better input image.

Another similar approach used two images, one is random image which is named as a reference image and the second is an input image. The reference image is used to correct the input image. Gaussian filter is used to address the blurring problem. After that, the algorithm uses the kernel density function to minimize the energy function and generate the lookup table for each colour channel independently in order to enhance the images [18].

Lateral inhibition of the neurons in the brain and Weber-Fechner law are two phenomenons. These two theories have been combined for contrast enhancement of an image [19]. Later on, Inverse Weber-Feahner function is proposed to enhance the colour images which are affected by contrast [20]. This approach is unable to improve the quality of the images. Furthermore images become blur after using their method.

In order to achieve better results, the original histogram of an image is used to apply the Maximum Likelihood Gamma Distribution (MLGD) method to get the midpoints of the histogram in order to divide the histogram into two modes. Then, the histogram is stretched based on these two modes; in other words, the histogram is stretched to two sides (i.e., minimum and maximum). This method has been applied to enhance the bimodal MRI (Magnetic Resonance Imaging) medical images [21]. One of the characteristics of this method is its simplicity. This method has only been used to enhance the gray images. The drawback is that it is unable to remove the colour cast as this method intends to stretch both side values.

In another approach, the researchers integrated the contrast enhancement method with the gamma correction method. Firstly, they normalize two images based on the histograms and then they combine these images with contrast enhancement image [22]. Gamma correction method has also been proposed to address the illumination problem [23]. This method uses a reference image to enhance the input image. The underlying algorithm of this method relies on the reference image to find the best illumination solution to address the illumination problem in order to enhance the input image.

To address the problem of illumination, pose and face expression, [24] have proposed a method that can create 3D face image based on 2D face image. As 3D face images are highly dependent on quality of $2 \mathrm{D}$ images. Therefore in their method they have used image enhancement method that can enhance 2D face images in order to create better 3D face image for recognition purposes.

They [25] have proposed a method that uses intensity range of an image in order to enhance the colour image which suffers from illumination due to sensor or distance (distance between camera and object). The B-spline interpolation method is used to control low $\mathrm{S} / \mathrm{N}$ (signal to noise ratio) of intensity range. Lastly luminance estimation method is used in order to compensate the illuminated colour.

[26] have used several image enhancement methods, such as gray world, white patch, gray edge, to improve the quality of the images. The approach uses the content based image retrieval rules for classification of suitable image enhancement method. 
This determines which image enhancement method needs to be applied to improve the quality of a particular image depending on the nature of that image. The selection of a suitable algorithm is made based on the estimation of an illumination using forest tree approach that is based on CART methodology.

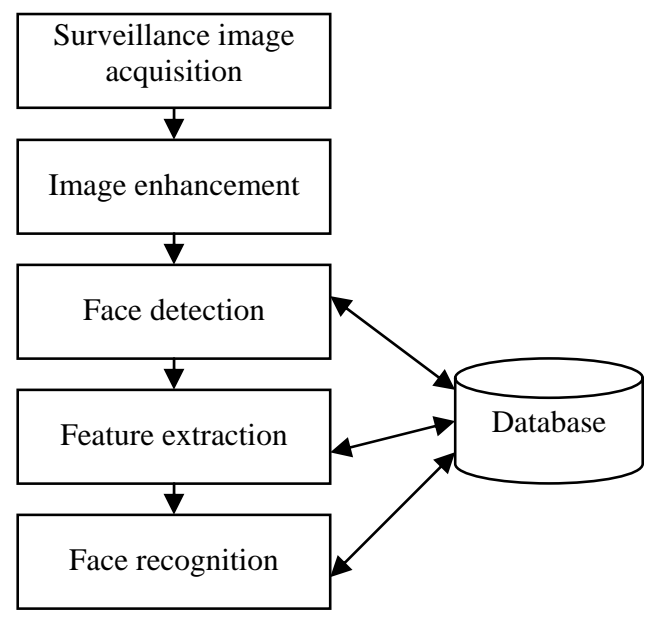

Figure 1: An Integrated Framework

\section{PROPOSED FRAMEWORK}

Biometrics security is concerned with identifying humans based on their unique physical and behavioural traits. By physical traits we mean the feature of human body such as fingerprint

This section discusses an integrated framework for biometric security. The framework emphasises the use of different image enhancement techniques that can improve face detection, feature extraction and face recognition for biometric security. The framework consists of three stages:

- At the first stage, image enhancement techniques such as contrast enhancement, colour balancing and gamma correction methods are applied to improve the visibility of an image which is important for face detection.

- At the second stage, image enhancement methods, noise removal, edge detection and feature selection method are used for feature extraction.

- At the third stage, AI methods such Bayesian and Neural network are used to compare the images with the stored images for feature matching and face recognition.

The proposed framework will be applied to images produced by surveillance cameras which usually produce low quality images. The main advantage of proposed framework is to improve the efficiency and accuracy of biometric security system by applying image enhancement techniques.

The components of the proposed framework are discussed below:

\subsection{Image enhancement}

In order to apply image enhancement techniques the surveillance videos are divided into a set of frames or images.
Surveillance cameras usually produce low quality videos. Therefore in the proposed framework, multiple methods of image enhancement are proposed in order to increase the contrast, make the image balanced and improve the illumination of an image. The following image enhancement methods are used to improve the quality of images in order to improve efficiency and accuracy of biometric system.

In order to improve the visibility of the surveillance images three image enhancement methods namely contrast enhancement, colour balancing and gamma correction methods are applied to:

- Improve the low contrast surveillance image by using contrast enhancement method.

- $\quad$ Balance the values of Red, Green and Blue components of a RGB colour image in order to produce a well balanced image and adjust the dominant colour in the rest of an image.

- Improve the brightness of low illuminated image by using gamma correction method.

\subsection{Face detection}

The enhanced images are used to detect objects, such as human face, to be used for further processing for face recognition purposes. The advantage of this stage is to detect the face and process that part of the image only in order to save computational resources that might be required to process the whole image.

Image and feature based methods normally used for face detection. The proposed framework takes advantage of both categories of face detection methods. Image based techniques normally rely on machine learning and statistical analysis. These techniques are trained based on the images rather than the abstract level information about the features of face. These techniques include Bayesian network and Neural Network.

Feature based approaches rely on skin colour or edges or global face knowledge. Skin colour is also commonly used to detect faces. In this method face detection is achieved by firstly applying colour segmentation methods for image segmentation purposes. These methods segment the image based on their colours that eventually help to detect the face based on their skin colour [27].

The enhanced images are used as input to the face detection methods in order to detect the faces. The existing face detection methods produce poor results when applied to the surveillance images. After the enhancement of surveillance images, these methods can produce better results.

\subsection{Feature extraction}

In pattern recognition the feature extraction stage plays a vital role. The pattern recognition is a method to identify objects based on their features extracted from their images which are then used to classify them based on statistical information or priori knowledge. The classified data or patterns are used to train the system for supervised as well as unsupervised learning. In unsupervised method, no information is given to the system but the system creates patterns based on its built-in functions. Normally statistical and syntactic (rule based) methods are used for pattern recognition. 
In this stage the features of faces are extracted. Feature extraction is important for face recognition as the whole process of recognition is highly dependent on the features of an image. Thus a suitable method will ensure better results are achieved for face recognition. The following feature extraction methods will be further investigated.

- $\quad$ Noise removal methods: Mean and median filter will be used for this stage.

- $\quad$ Edge detection of face images: Sobel and Canny edge detection method will be thoroughly explored, and suitable method will be selected to use as part of the proposed framework.

- Edge thinning: Edges that are taken through edge detection method will be further processed using edge thinning algorithms in order to get accurate edges which will be used for feature extraction.

- $\quad$ Feature selection and extraction: In this step features will be selected and used for feature extraction method. Feature matching will be performed based on the selected features for face recognition.

\subsection{Face recognition}

The features extracted in the previous stage are used for feature matching. The features of the face are matched with the reference images that are taken from the database. Statistical methods are used to find the nearest neighbouring features based on the highest probability feature. AI methods such as Bayesian and Neural network, k-means and k-d tree algorithms will be investigated for this purpose and a suitable method will be used. Face recognition cannot be improved without improving the quality of images [5]. Thus an important part of the proposed approach is to use image enhancement to enhance the quality of the images in order to improve the efficiency and accuracy of biometric security system.

\section{CONCLUSION}

In this paper, we have proposed an integrated framework consisting of four components namely image enhancement, face detection, feature extraction and face recognition. Additionally, several methods are discussed that would be used for each stage of the proposed framework. An important aspect of face recognition is to apply image enhancement methods that can significantly improve the quality of the images and make them ready for face recognition. This is particularly important to apply the image enhancement techniques to low quality images produced by surveillance cameras. It is envisaged that the proposed integrated framework will produce better results when applied to surveillance images for biometric security.

\section{REFERENCES}

[1] Anil K. Jain, Arun Ross and Salil Prabhakar.2004. An introduction to biometric recognition. IEEE Transactions on Circuits and Systems for Video Technology, Volume 14, Issue 1.

[2] Raj Mudigoudar, Srinath Bagal, Zhanfeng Yue, Pramod Lakshmi and Pankaj Topiwala. 2009. Video superresolution: from QVGA to HD in real-time. Applications of
Digital Image Processing XXXII. Proceedings of the SPIE, Volume 7443, Pages 74430W-74430W-12

[3] Mark Smids. 2006. Background Subtraction for Urban Traffic Monitoring using Webcams. Master Thesis, Universiteit van Amsterdam, FNWI.

[4] Abdullah C. and Salih G. 2008. A Fast Fingerprint Image Enhancement Algorithm Using a Parabolic Mask. Computers and Electrical Engineering, Volume 34, Issue 3, Pages 250256, Elsevier Science.

[5] J. Wang, C. Zhang, and H.-Y. Shum. 2004. Face image resolution versus face recognition performance based on two global methods. In Proceeding of Asian Conference on Computer Vision.

[6] Shaokang Chen, Erik Berglund, Abbas Bigdeli, Conrad Sanderson, and Brain C. Lovell. 2008. Experimental analysis of face recognition on still and cctv images. In Proceedings of IEEE International Conference on Advanced Video and Signal Based Surveillance, Pages 317-324.

[7] Vahid Abolghasemi and Alireza Ahmadyfard. 2009. An edge-based color-aided method for license plate detection. Image and Vision Computing, Pages 1134-1142, Elsevier Science.

[8] Danian Zheng, Yannan Zhao and Jiaxin Wang. 2005. An efficient method of license plate location. Pattern Recognition Letters, 26, Pages 2431-2438, Elsevier Science

[9] Liangpei Zhang, Hongyan Zhang, Huanfeng Shen and Pingxiang Li. 2010. A super-resolution reconstruction algorithm for surveillance images. Signal Processing, Elsevier Science.

[10] Chanyul Kim and Noel E.O’Connor, 2009. Using the discrete hadamard transform to detect moving objects in surveillance video. International Conference on Computer Vision Theory and Applications, Porto, Portugal,

[11] Tao Yang, Stan Z. Li, Quan Pan, and Jing Li. 2004. Realtime and accurate segmentation of moving objects in dynamic scene. In ACM International Workshop on Video Surveillance and Sensor Networks.

[12] Pakorn KaewTrakulPong and Richard Bowden. 2003. A Real-Time Adaptive Visual Surveillance System for Tracking Low-Resolution Colour Targets in Dynamically Changing Scenes. Image and Vision Computing, Volume 21, Pages 913-929, Elsevier Science.

[13] Barry Ahrens. 2005. Genetic Algorithm Optimization of Superresolution Parameters. In Proceedings of the 2005 Conference on Genetic and Evolutionary Computation.

[14] Yutaka Hatakeyama, Kazuhiko Kawamoto, Hajime Nobuhara, Shin-ichi Yoshida and Kaoru Hirota. 2005. Color restoration algorithm for dynamic images under multiple luminance conditions using correction vectors. Pattern Recognition Letters, Volume 26 Issue 9, Pages 1304-1315, Elsevier Science.

[15] Wen-Chung Kao, Ming-chaihsu and Yueh-yiingyang. 2009. Local contrast enhancement and adaptive feature extraction for illumination-invariant face recognition. Pattern Recognition Letters, Elsevier Science. 
[16] Gaoyun An, Jiying Wu and Qiuqi Ruan. 2010. An illumination normalization model for face recognition under varied lighting conditions. Pattern Recognition Letters, Volume 31, Pages 1056-1067, Elsevier Science.

[17] Youngsung Kim, Andrew Beng Jin Teoh and Kar-Ann Toh. 2010. A performance driven methodology for cancelable face templates generation. Pattern Recognition, Volume 43, Pages 2544-2559, Elsevier Science.

[18] Mehrdad Panahpour Tehrani, Akio Ishikawa, Shigeyuki Sakazawa and Atsushi Koikea. 2010. Iterative colour correction of multicamera system using corresponding feature points. Journal of Visual Communication and Image Representation, Volume 21, Issues 5-6, Pages 377-391, Elsevier Science.

[19] Kobayashi,Y., Kato,T., and Ohya,J. 2007. A contrast enhancement method by reverse-s shape transformation. IEICE Trans. Inf. Syst. (Jpn. Ed.) J90-D, Pages 1263-1274.

[20] Kazushi Murakoshi and Mai Miura. 2010. Image correction method for colour contrast affect using inverse processes of the brain. BioSystem, (in press). Elsevier Science.

[21] Al-Manea, A. and El-Zaart, A. 2007. Contrast enhancement of MRI images. Lecture Notes in Computer Science (LNCS) Springer, ISBN 978-3-540-68016-1, pp, 255-258.

[22] Zhi-Guo Wang, Zhi-Hu Liang, Chun-Liang Liu. 2009. A real-time image processor with combining dynamic contrast ratio enhancement and inverse gamma correction for PDP.
Displays Volume 30, Issue 3, Pages 133-139 , Elsevier Science.

[23] Alexander Wong and William Bishop. 2007. Simultaneous Gamma Correction and Registration in the Frequency Domain. International Conference on Image Processing, Computer Vision \& Pattern Recognition, Pages 145-151, Las Vegas Nevada, USA.

[24] A.B. Moreno, A. Sánchez , E. Frías-Martínez and J.F. Vélez. 2010. Three-dimensional facial surface modeling applied to recognition. Engineering Applications of Artificial Intelligence, Volume 22, Issue 8, Pages 1233-1244, Elsevier Science.

[25] Megumi Shinozaki, Masato Kusanagi, Kazunori Umeda, Guy Godin and Marc Rioux. 2009. Correction of color information of a 3D model using a range intensity image. Computer Vision and Image Understanding, Volume 113, PP 1170-1179, Elsevier Science.

[26] S. Bianco, G.Ciocca, C.Cusano and R.Schettini. 2010. Automatic Color Constancy Algorithm Selection and Combination. Pattern Recognition, Volume 43, Pages 695705, Elsevier Science.

[27] Jose M. Chaves-González, Miguel A. Vega-Rodríguez, Juan A. Gómez-Pulido, Juan M. Sánchez-Pérez. 2010. Detecting skin in face recognition systems: A colour spaces study. Digital Signal Processing, Volume 20, Pages 806-823, Elsevier Science. 